Radiation-hard ASICs for optical data transmission in the first phase of the LHC upgrade

This article has been downloaded from IOPscience. Please scroll down to see the full text article.

2010 JINST 5 C12006

(http://iopscience.iop.org/1748-0221/5/12/C12006)

View the table of contents for this issue, or go to the journal homepage for more

Download details:

IP Address: 137.138.124.142

The article was downloaded on 11/07/2011 at 10:19

Please note that terms and conditions apply. 


\title{
Radiation-hard ASICs for optical data transmission in the first phase of the LHC upgrade
}

K.K. Gan, ${ }^{a, 1}$ P. Buchholz, ${ }^{b}$ H.P. Kagan, ${ }^{a}$ R.D. Kass, ${ }^{a}$ J.R. Moore, ${ }^{a}$ D.S. Smith, ${ }^{a}$ A. Wiese ${ }^{b}$ and M. Ziolkowskic ${ }^{b}$

\author{
${ }^{a}$ Department of Physics, The Ohio State University, \\ 191 W. Woodruff Ave., Columbus, OH 43210, U.S.A. \\ ${ }^{b}$ Fachbereich Physik, Universität Siegen, \\ Siegen, Germany \\ E-mail: gan@mps.ohio-state.edu
}

\begin{abstract}
We have designed two ASICs for possible applications in the optical links of a new layer of the pixel detector to be install inside the ATLAS Pixel detector for the first phase of the LHC luminosity upgrade. The ASICs include a high-speed driver for the VCSEL and a receiver/decoder to decode the signal received at the PIN diode to extract the data and clock. Both ASICs contain 4 channels for operation with a VCSEL or PIN array. The ASICs were designed using a $130 \mathrm{~nm}$ CMOS process to enhance the radiation-hardness. We have characterized the fabricated ASICs and the performance of the ASICs is satisfactory. The receiver/decoder can properly decode the bi-phase marked input stream with low PIN current and the driver can operate a VCSEL up to $\sim 5 \mathrm{~Gb} / \mathrm{s}$. The added functionalities are also successful, including redundancy to bypass a broken VCSEL or PIN channel, individual control of VCSEL current, and power-on reset circuit to set all VCSEL currents to a nominal value. The ASICs were irradiated to a dose of $46 \mathrm{Mrad}$ with $24 \mathrm{GeV} / \mathrm{c}$ protons. The observed modest degradation is acceptable and the single event upset rate is negligible.
\end{abstract}

KEYWORDS: Optical detector readout concepts; Radiation-hard electronics

\footnotetext{
${ }^{1}$ Corresponding author.
} 


\section{Contents}

1 Introduction 1

2 DORIC 1

3 VDC 3

4 Radiation hardness $\quad 4$

5 Summary 6

\section{Introduction}

The Large Hadron Collider (LHC) at CERN, Geneva, Switzerland, will be upgraded in two phases to increase the luminosity. The ATLAS experiment plans to add a new pixel layer to the current pixel detector during the first phase of the upgrade. The optical data transmission will also be upgraded to handle the high data transmission speed. The upgrade for the on-detector optical links will be based on VCSEL and PIN arrays operating at $850 \mathrm{~nm}$ as in the current system. Two ASICs have been designed for this new generation of optical links to incorporate the experience gained from the current system.

The ASICs [1] ${ }^{1}$ have been designed using $130 \mathrm{~nm}$ 8RF-DM CMOS process. The driver couples to a VCSEL array with one channel designated as a spare. Similarly for a receiver/decoder that couples to a PIN array. With the inclusion of a remote control interface, this allows redundancy in both directions by enabling a signal to be re-routed from a bad VCSEL or PIN channel. The submitted chip contains four VCSEL drivers (VDC), four PIN diode receivers/decoders (Digital Opto-Receiver Integrated Circuit or DORIC), and the associated circuitry to control the re-routing of the signals to the designated spare channels. All circuitry within the test chip was designed following test results and guidelines from CERN on radiation tolerant design in the $130 \mathrm{~nm}$ process used [2]. We have characterized the fabricated chips and then irradiated the chips to a dose of 46 Mrad using $24 \mathrm{GeV} / \mathrm{c}$ protons. The preliminary results of the study will be presented below.

\section{DORIC}

Each of the DORIC contains a PIN diode receiver, a bi-phase marked (BPM) clock/data recovery circuit, and has low voltage differential signal (LVDS) outputs for both the clock and data. All four DORICs are designed to operate with a $1.5 \mathrm{~V}$ power supply and a $40 \mathrm{Mb} / \mathrm{s}$ BPM input stream. One of the DORICs includes circuitry to extend its operational speed to 80,160 , or $320 \mathrm{Mb} / \mathrm{s}$. Twochannel multiplexers have been inserted in the post amplification paths of the non-spare DORIC

\footnotetext{
${ }^{1}$ The VCSEL driver and PIN receiver is an updated version of the chips designed in the $250 \mathrm{~nm}$ process.
} 


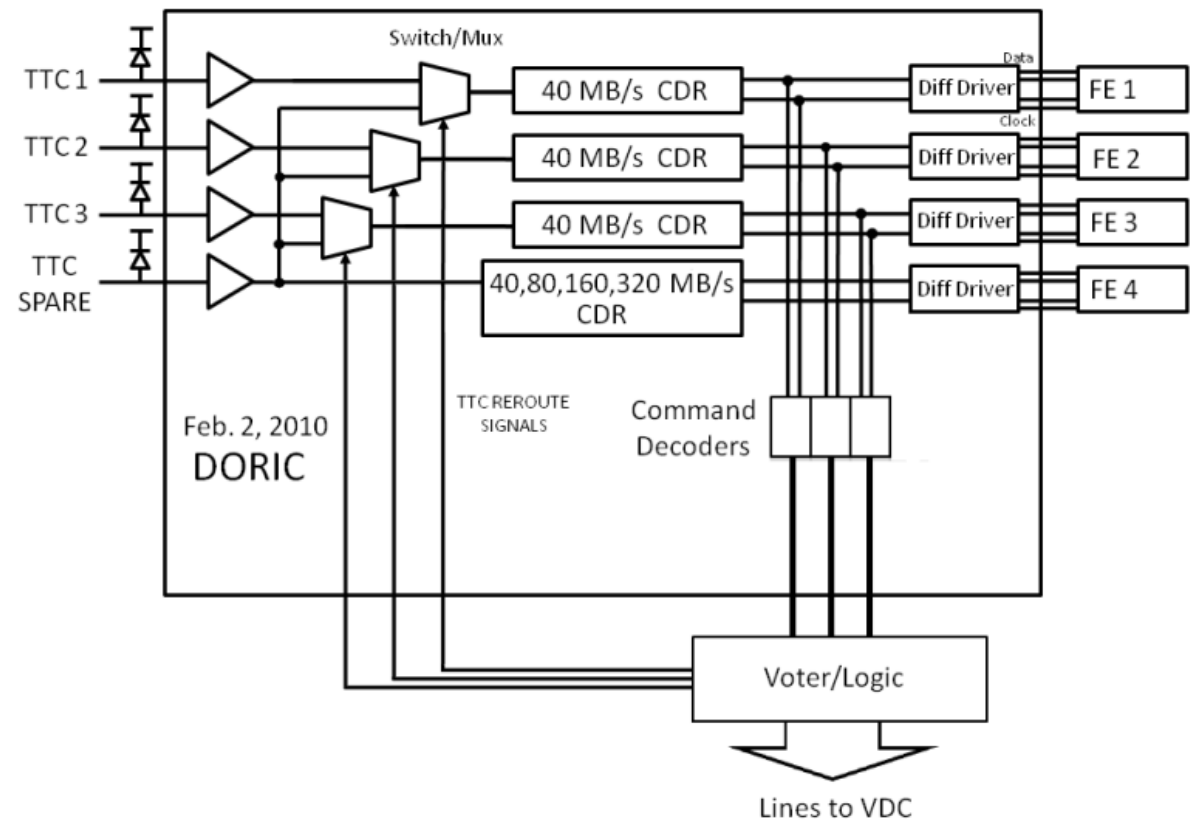

Figure 1. DORIC block diagram. In the final design, there will be one less output than input, i.e. if one of the PIN diode malfunctions, the optical signal will be transmitted via the spare PIN to the original FE channel.

channels. With these multiplexers, the signal from the spare amplifier channel may be routed to the channel of choice. A block diagram of the DORIC section of the chip is shown in figure 1.

In order to allow remote control over channel steering and other functionality within the chip, a command decoder has been included in three of the DORICs. The command decoder was designed for the front-end (FE) chips of the new pixel layer. Because the chips will be resided in a high radiation environment, special care was taken to improve the single event upset (SEU) tolerance of the command decoder. The command word for configuring the chip is formed by a majority vote of the three command decoders. To further improve our chip's tolerance to SEUs, all latches in our design are based on a dual interlocked storage cell (DICE) latch designed for use in the configuration memory of the front-end chips of the pixel detector.

To simplify some of the testing and to allow verification of the command decoder's operation, we have included a serially interfaced test port to the command word. Figure 2 shows a block diagram of the interface.

All four DORIC channels can properly decode the data at $40 \mathrm{Mb} / \mathrm{s}$ with no bit errors for low input PIN currents, $\sim 20 \mu \mathrm{A}$ for the three single-speed channels and $\sim 40 \mu \mathrm{A}$ for the multispeed channel. For the multi-speed channel, the threshold is higher at higher speed, from $\sim 60$ $\mu \mathrm{A}$ at $80 \mathrm{Mb} / \mathrm{s}$ to $\sim 100 \mu \mathrm{A}$ at $320 \mathrm{Mb} / \mathrm{s}$. The cause for the higher thresholds is currently under investigation. The clock jitter (peak-to-peak) at $40 \mathrm{Mb} / \mathrm{s}$ is $\sim 1.4 \mathrm{~ns}$ for both single-speed and multi-speed channels. For the multi-speed channel, the jitter is smaller at higher operating speed, as little as $\sim 100 \mathrm{ps}$ at $320 \mathrm{Mb} / \mathrm{s}$. It should be noted that the multi-speed channel requires external bias tuning for proper operation at 160 and $320 \mathrm{Mb} / \mathrm{s}$ due to the limited dynamic range of the 


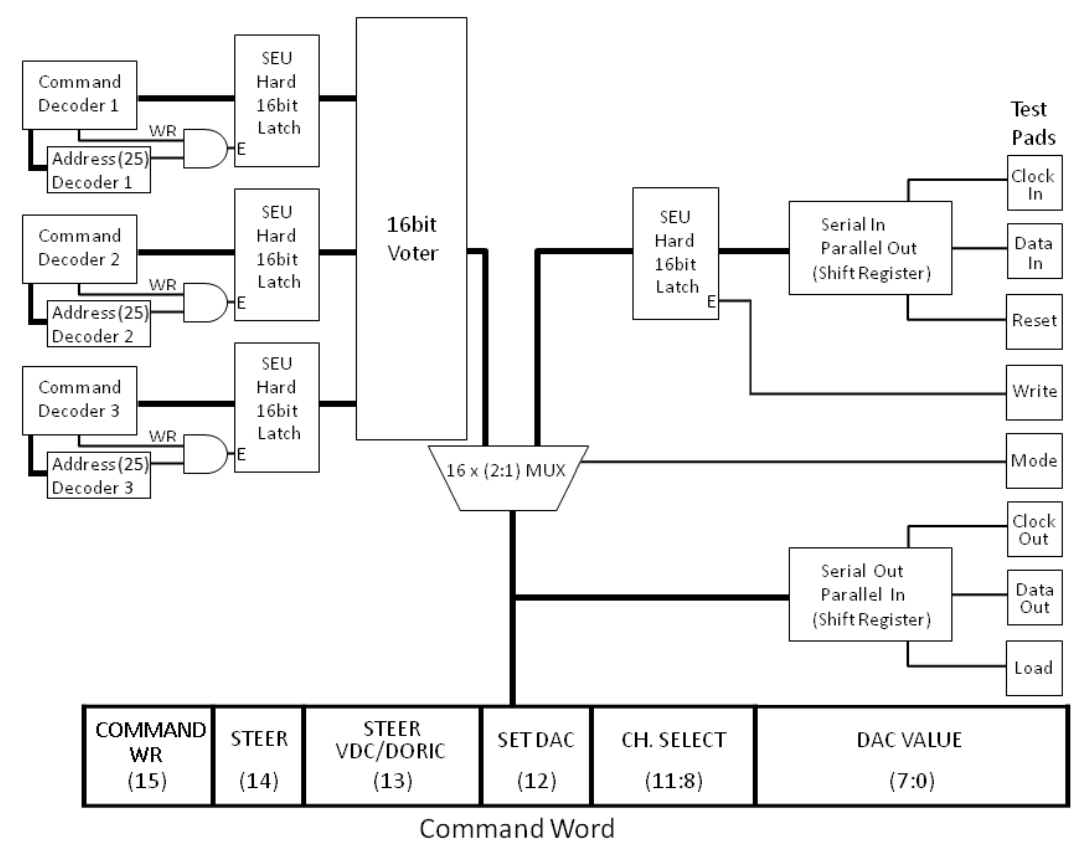

Figure 2. There are two interfaces for loading the 16-bit word needed for the ASIC operation: the command decoder interface on the left or the test port on the right.

clock duty cycle control circuitry and further improvement is needed in the next submission. The steering circuit functions properly, i.e. signal received at the spare DORIC channel can be rerouted through the other DORIC channels. However, this can only be excised via the test port because the scan chain enable of the command decoder was left floating due to a miscommunication with the designer of the command decoder.

\section{VDC}

The four VDC channels are designed to operate at speeds up to $5 \mathrm{~Gb} / \mathrm{s}$. Each channel has an LVDS receiver, an 8-bit DAC, and a driver stage for converting the received LVDS signal into a current sufficient to drive a VCSEL. Two of the four VDC channels provide the capability to add a pre-emphasis current to the VCSEL modulation current. The duration of the pre-emphasis current pulse and its amplitude are adjustable via wire-bond pads. One of the VDC channels is designated as the spare channel and contains a 16:1 multiplexer. The multiplexer allows routing of the received LVDS signal from any of the other three channels to the spare channel output. A block diagram of the VDC portion of the test chip is shown in figure 3.

The 8-bit DAC and LVDS receiver are designed to operate with a $1.5 \mathrm{~V}$ supply and the driver stage is designed to operate with a $2.5 \mathrm{~V}$ power supply. A higher supply voltage is required by the output stage to allow enough headroom to drive VCSELs which normally have threshold voltages of $\sim 2 \mathrm{~V}$. The output driver is capable of delivering up to $12 \mathrm{~mA}$ modulation current and up to 4 $\mathrm{mA}$ bias current if driving a VCSEL with a $2 \mathrm{~V}$ threshold voltage. 


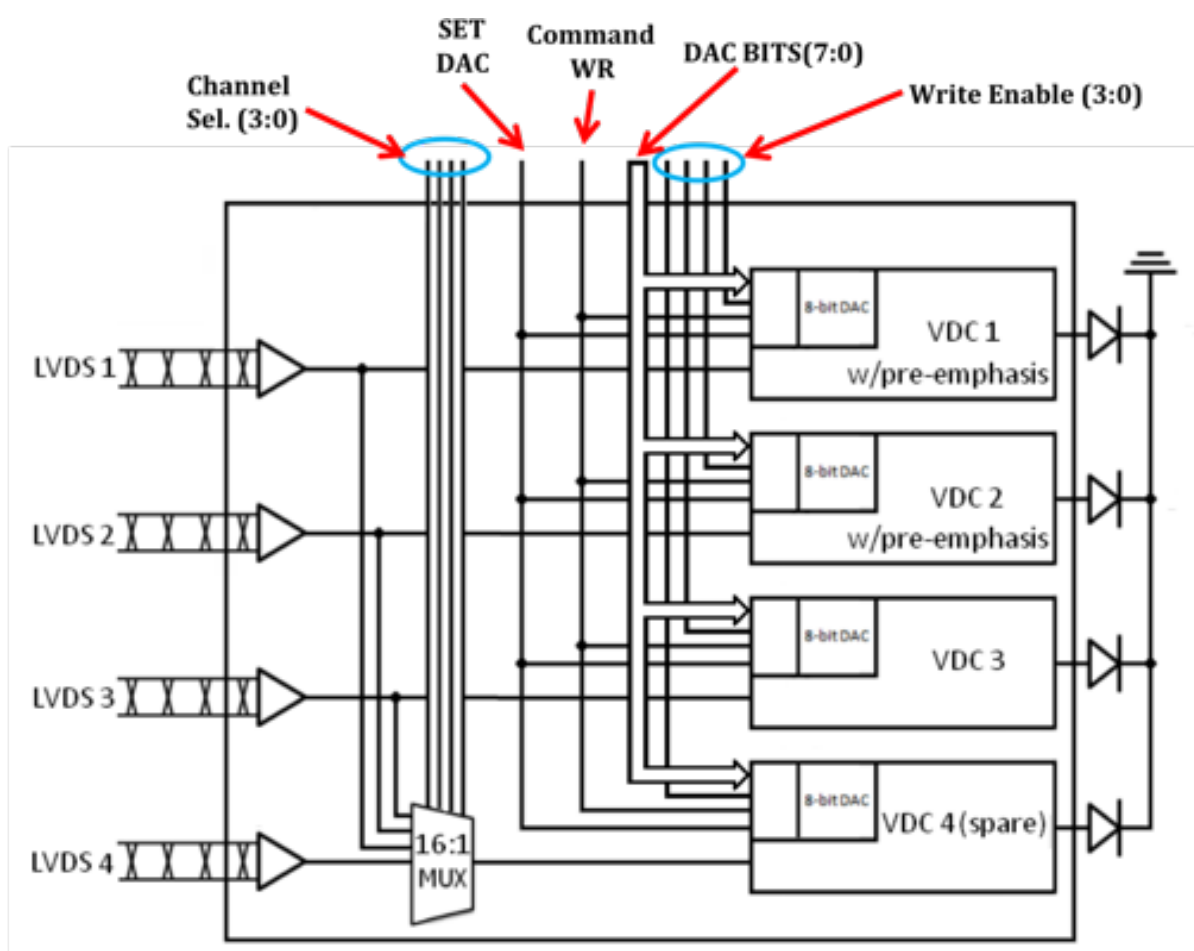

Figure 3. VDC block diagram. In the final design, there will be one less input than output, i.e. if one of the VCSELs malfunctions, the LVDS signal will be transmitted via the spare VDC/VCSEL.

The 8-bit DAC is used to set the VCSEL modulation current. The setting of each DAC is accomplished by sending appropriate commands to the command decoders in the DORIC. To enable operation in case of a failure in the communication link to the command decoder, we have included a power-on reset circuit that will set the VCSEL modulation current to $10 \mathrm{~mA}$ upon power up. Furthermore, we have included a test mode that allows bypassing of the DAC to directly set the modulation current via a wire bond pad. This functionality will not be implemented in final design.

In the prototype VDC, the power-on reset circuit functions properly, supplying a VCSEL modulation current of $\sim 10 \mathrm{~mA}$ upon power up. We can steer the signal received via the test port to the spare VDC/VCSEL. In addition, we can set the DAC to control individual VCSEL currents. All four channels run error free at $5 \mathrm{~Gb} / \mathrm{s}$ with bit error rate of $<5 \times 10^{-13}$, including the spare channel with the signal routed from the other LVDS inputs. Figure 4 shows the eye diagrams of the optical signal in one channel and the signal routed from a spare channel. It is evident that the eye is "open" but more improvement is desirable for better operating margin.

\section{Radiation hardness}

Two chips were packaged for irradiation with $24 \mathrm{GeV} / \mathrm{c}$ protons at CERN in August 2010 to evaluate the radiation hardness of the devices. Each chip contained 4 channels of drivers and receivers. The total dose received was $1.7 \times 10^{15}$ protons $/ \mathrm{cm}^{2}$ (46 Mrad). All testing were electrical to avoid complications from degradation of optical components. The remote location required the use of long cables which limited the testing to low speed. We observed little degradation of devices. 

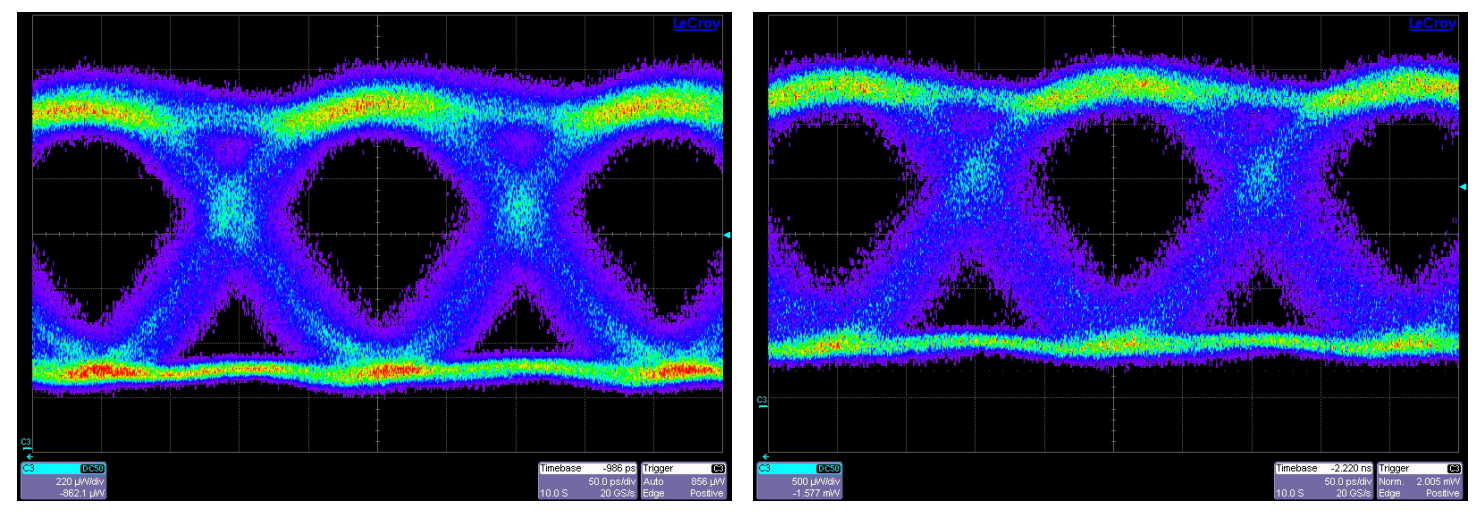

Figure 4. Eye diagram of the optical signal in a VDC channel (left) and the signal routed via a spare channel (right). The signal is measured with a $4.5 \mathrm{GHz}$ optical probe.

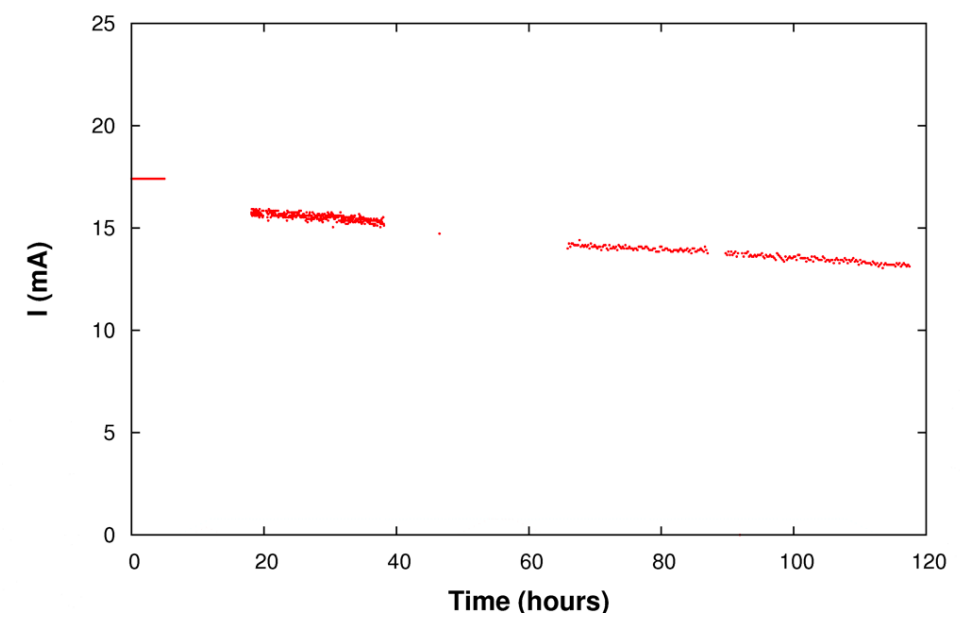

Figure 5. Current in the $25 \Omega$ resistor driven by a VDC as a function of time during the irradiation. The dose at the end of the irradiation is $1.7 \times 10^{15}$ protons $/ \mathrm{cm}^{2}$.

In the 2008 irradiation with the previous version of the VDC, we observed significant decrease in the current consumption and the output drive current. The degradation was due to the fact that the current mirror in the driver circuit used thick oxide technology with a mix of PMOS and NMOS transistors in order to produce sufficient voltage to drive a VCSEL. Consequently the circuit was sensitive to the different threshold shifts of the NMOS and PMOS after irradiation, which is larger in the NMOS than in the PMOS. We used PMOS transistors only in the present design and the decrease in the drive current is now quite modest (figure 5).

There are a total of 126 SEU hardened latches per 4-channel chip which could be upset by traversing particles. During irradiation we monitored both the amplitude of the VDC drive currents and the signal re-routing functions for possible SEU. We observed 13 occurrences of an error in these functionalities during 71 hours of irradiation in one chip and a similar SEU rate in the other. This corresponds to a SEU cross section of $\sim 1 \times 10^{-16} \mathrm{~cm}^{2}$. The particle flux is $\sim 3 \times 10^{9}$ $\mathrm{cm}^{-2} /$ year at the opto-link location. This implies a SEU rate of $\sim 3 \times 10^{-7} /$ year/link. Obviously this is quite acceptable. 


\section{Summary}

We have successfully fabricated 4-channel driver and receiver ASIC arrays in the $130 \mathrm{~nm}$ CMOS process for use in the optical link upgrade. The receiver properly decodes the bi-phase marked input stream with low PIN current and the driver can operate a VCSEL up to $\sim 5 \mathrm{~Gb} / \mathrm{s}$. The added functionalities are also successful, including redundancy to bypass a broken VCSEL or PIN channel, individual control of VCSEL current, and power-on reset circuit to set all VCSEL currents to a nominal value. The ASICs were irradiated to a dose of $46 \mathrm{Mrad}$ with $24 \mathrm{GeV} / \mathrm{c}$ protons. The observed modest degradation is acceptable and the SEU rate is negligible. We plan to submit a 12-channel version incorporating the lesson learned from this submission.

\section{Acknowledgments}

The authors are indebted to Maurice Glaser for his tireless effort to help in using the T7 irradiation facility at CERN. This work was supported in part by the U.S. Department of Energy under contract No. DE-FG-02-91ER-40690 and by the German Federal Minister for Research and Technology (BMBF) under contract No. 056Si74.

\section{References}

[1] K. Arms et al., ATLAS pixel opto-electronics, Nucl. Instrum. Meth. A 554 (2005) 458.

[2] F. Faccio, personal communication (2007). 